IRSTI 29.05.27

\title{
The fundamental equation of the field theory in De Sitter pulse space
}

\author{
R.M. Ibadov* and S.N.Murodov \\ Department of Theoretical Physics, Samarkand State University, \\ University blv. 15, 140104, \\ Samarkand, Republic of Uzbekistan \\ *e-mail: ibrustam@mail.ru
}

\begin{abstract}
The fundamental equation of the field theory in De Sitter pulse 5-dimensional space is obtained. The wave function, subordinated to this equation in usual space-time, doubles. One of these functions, probably to consider as the candidate on "the Phantom field" responsible for expansion of the Universe with acceleration. The cross sections of $e^{-} e^{-} \rightarrow e^{-} e^{-}, e^{-} e^{+} \rightarrow e^{-} e^{+}$and $e^{-} e^{+} \rightarrow \mu^{-} \mu^{+}$processes at high energies calculated taking into account polarized initial and final particles. Is estimated contribution FM to cross-section sections. Some experimental consequences are predicted. In all these calculations the polarization of particles be taken into account. The Lagrangian of quantum electrodynamics with the fundamental mass is chosen as an effective interaction Lagrangian. All the calculations are made in the Euclidian space, the transfer to the ordinary pseudoeuclidian space is established in the final expressions only. The approach has been based on the assumption that the momentum space possesses the geometric structure of a de Sitters pace of constant curvature. A key role has been be assigned to this constant radius of curvature.
\end{abstract}

Key words: field theory, De Sitter pulse, cross sections, quantum field theory.

PACS numbers: 10, 04.20.-q, 03.67.Lx, 04.62. + V, 02.30.Ик

\section{Introduction}

This work continues earlier researches of the academician of Kadyshevsky V.G. and its pupils $[1,2]$ towards constructing a consistent new Quantum Field Theory (QFT) with fundamental mass (FM) $M$, defining a hypothetical but universal scale in the region of ultrahigh energies. From a theoretical point of view the fundamental mass $M$ and corresponding the fundamental length $\ell=$ $\hbar / M c$ are supposed to play a major role such as Planck's constant $\hbar$, the speed of light $c$ or Newton's gravitational constant $\mathcal{H}$. The existence of so-called ultra-violet divergences, i.e., infinitely large values, arising as a result of direct application of equations QFT in area of very small space-time distances, or equivalently, to the region of very high energies and pulses is one of lacks of standard QFT. There were ideas about presence of a new universal constant dimension of mass or length in nature $[3,4,5,6]$, which would fix the certain scale in the field of high energies or on small space-time distances because of the purpose to give the decision of this problem in the most various contexts. They testify only that the modern high-energy physics still far will defend from that boundary behind which can be shown new geometrical properties of space-time. From a position of today it is represented to many theorists rather probable, that the "true" theory of the field, capable to give the adequate description of all interactions of elementary particles, will be at least renormalized by Lagrange theory having local gauge supersymmetry. It is asked, whether such scheme can contain such a parameter as the fundamental length? The future experiments can give the answer to this question only. However, numerous attempts to construct more general QFT, proceeding from such parcels, did not give essential results. This failure is probable speaks that for today the mathematical theory of spaces which geometry only "in small" differs from (pseudo) Euclidean geometry is not developed almost and, especially, in similar kinds of spaces the mathematical device adequate to requirements QFT is not advanced. But, the output from the created situation is prompted by QFT. As it is known, within the framework of this 
theory space-time description is completely equal in rights with the description in terms pulse-power variables. If the theory is formulated in pulse representation fields, sources, Green's functions and other attributes of the theory appear certain in fourdimensional (pseudo) Euclidean p-space. This modified quantum field theory has an elegant geometrical basis: in momentum representation one faces a momentum space corresponding to de Sitter space of curvature radius $[1,2,7,8]$. The approach developed earlier has been based on the assumption that the momentum space possesses the geometric structure of a de Sitters pace of constant curvature. A key role has been be assigned to this constant radius of curvature.

\section{Theory}

Experimental Detection of the new fundamental scale testifying to existence specific atoms of spacetime, would mean, that in knowledge of a nature the new step, commensurable on the value with opening quantum properties of a matter is made. According to modern data, if the constant $\ell$ also exists then if submits to restriction $\ell \leq 10^{-19} \mathrm{~cm}$. This boundary still extremely far will defend from "Planck lengths" $\ell_{\text {Planck }} \sim 10^{-33} \mathrm{~cm}$, determining spatial scales of effects of quantum gravitation. And, certainly, it is impossible to exclude, that in process of overcoming an enormous interval $10^{-19}-10^{-33} \mathrm{~cm}$ will be open the new physical phenomena and laws, associate with new "scale of a nature" fundamental length $\ell$.

In the papers cited [9], the key role was played by the following geometric idea: to construct QFT providing an adequate description of particle interactions at super high energies, one should write down the standard field theory in the momentum representation, and then pass it from the Minkowski $\mathrm{p}$-space to the de Sitter p-space with a large enough radius.

The de Sitter space has a constant curvature. Depending on its sign there are two possibilities

$$
\begin{gathered}
p_{0}^{2}-p_{1}^{2}-p_{2}^{2}-p_{3}^{2}+p_{5}^{2} \equiv g^{K L} P_{K} P_{L}=M^{2} ; \\
K, L=0,1,2,3,5 \\
\text { (positive curvature: } g^{00}=-g^{11}=-g^{22}= \\
\left.-g^{33}=g^{55}=1\right) \\
p_{0}^{2}-p_{1}^{2}-p_{2}^{2}-p_{3}^{2}-p_{5}^{2} \equiv g^{K}{ }^{\square} P_{K} P_{L}=-M^{2} ;
\end{gathered}
$$

$$
K, L=0,1,2,3,5
$$

(negative curvature: $g^{00}=-g^{11}=-g^{22}=$ $\left.-g^{33}=-g^{55}=1\right)$.

The non-Euclidean Lobachevsky imaginary 4space (2) is also called the Lobachevsky imaginary 4-space. It is natural that QFT based on momentum representation of the form (1)-(2) must predict new physical phenomena at energies $E \geq M$. In principle, the parameter may turn out to be close to the Planck mass

$$
M_{\text {Planck }}=\sqrt{\frac{\hbar c}{\kappa}} \approx 10^{19} \mathrm{GeV} .
$$

Then, the new scheme should include quantum gravity. The standard QFT corresponds to the "small" 4-momentum approximation

$$
\left|p_{0}\right|,|\vec{p}| \ll M, \quad p^{5}=g^{55} p_{5} \cong M,
$$

which formally can be performed by letting $M \rightarrow \infty$ (flat limit). Such features of the considered generalization of the theory as geometricity and minimality are intriguing. This is due to the fact that the Minkowski momentum 4-space having a constant zero curvature is a degenerate limiting form of each of the spaces with constant nonzero curvature (1)-(2).

If we substitute in (2) standard

$$
p_{\mu}=i \hbar \frac{\partial}{\partial x^{\mu}}
$$

and

$$
p_{5}=i \hbar \frac{\partial}{\partial x^{5}}
$$

receive the quantum version of the de Setter equations (2) in five-dimensional field equation

$$
\begin{gathered}
{\left[\frac{\partial^{2}}{\partial x^{\mu} \partial x_{\mu}}-\frac{\partial^{2}}{\partial x_{5}^{2}}-\frac{M^{2} c^{2}}{\hbar^{2}}\right] \Phi\left(x, x^{5}\right)=0,} \\
\mu=0,1,2,3 .
\end{gathered}
$$

We deliberately use in (3) the normal units to emphasize those three universal constants $\hbar, c$ and $M$ are grouped into one parameter-fundamental length $\ell=\hbar / M c$. Eq. (3) may be considered as the "fundamental" equation of motion. It is natural to 
extend the term "fundamental" to eq. (3) itself (for short f.e.). All the fields independent of their tensor (or spinor) character must obey eq. (3) since similar universality is inherent in the "classical" prototype, i.e. - de Sitter p-space (2). As applied to scalar, spinor, vector and other fields we shall write down the five-dimensional wave function $\Phi\left(x, x^{5}\right)$ in the form $\varphi\left(x, x^{5}\right), \psi_{\alpha}\left(x, x^{5}\right), A_{\mu}\left(x, x^{5}\right), \ldots$. The field theory based on f.e. (3) turns out to be more consistent and more general than the scheme developed in the de Sitter p-space (2). Thus, by virtue of (2) the 4-momentum components should obey the constraint

$$
p^{2}=p_{0}^{2}-\vec{p}^{2} \geq-M^{2},
$$

which does not follow from eq. (3). Indeed, passing in (3) to a mixed $\left(p, x^{5}\right)$ representation we get the equation

$$
\left[p^{2}+M^{2}+\frac{\partial^{2}}{\partial x_{5}^{2}}\right] \Phi\left(p, x^{5}\right)=0
$$

having a solution at all $p^{2}$ including the region $p^{2}+$ $M^{2}<0$. Consequently, $p^{5}=\sqrt{p^{2}+M^{2}}$ now takes both real and pure imaginary values. For further application let us define this quantity as a generalized function

$$
\begin{gathered}
p^{5}=\sqrt{p^{2}+m^{2}+i 0}= \\
=\left\{\begin{array}{l}
\sqrt{p^{2}+M^{2}}, \text { if } p^{2}+M^{2}>0 \\
i \sqrt{p^{2}+M^{2}}, \text { if } p^{2}+M^{2}<0
\end{array}\right.
\end{gathered}
$$

with (5) one can easily write down the general solution of (4)

$$
\begin{aligned}
\Phi\left(p, x^{5}\right)= & \cos \left(x^{5} \sqrt{p^{2}+m^{2}+i 0}\right) \Phi(p, 0)+ \\
& +\frac{\sin \left(x^{5} \sqrt{p^{2}+m^{2}+i 0}\right)}{\left.\sqrt{p^{2}+m^{2}+i 0}\right)} \frac{\partial \Phi(p, 0)}{\partial x^{5}}
\end{aligned}
$$

where the "initial data" of $\Phi(p, 0)$ and $\frac{\partial \Phi(p, 0)}{\partial x^{5}}$ are determined at all values of 4-momenta. The Fourier transformation of (6), results in the formal solution of fundamental equation (3)

$$
\begin{aligned}
& \Phi\left(x, x^{5}\right)=\frac{1}{(2 \pi)^{3 / 2}} \int e^{-i p x} d^{4} p \Phi\left(p, x^{5}\right)=\frac{1}{(2 \pi)^{3 / 2}} \int e^{-i p x} d^{4} p \times \\
& \quad \times\left\{\cos \left(x^{5} \sqrt{p^{2}+m^{2}+i 0}\right) \Phi(p, 0)+\frac{\sin \left(x^{5} \sqrt{p^{2}+m^{2}+i 0}\right)}{\left.\sqrt{p^{2}+m^{2}+i 0}\right)} \frac{\partial \Phi(p, 0)}{\partial x^{5}}\right\} .
\end{aligned}
$$

To compensate growing terms

$$
\operatorname{ch}\left(\sqrt{-p^{2}-M^{2}} x^{5}\right) \text { and } \operatorname{sh}\left(\sqrt{-p^{2}-M^{2}} x^{5}\right)
$$

and thus to give the meaning to integral (7), the arbitrary function $\Phi(p, 0)$ and $\frac{\partial \Phi(p, 0)}{\partial x^{5}}$ should obey at least the exponential conditions of decreasing in the region

$$
p^{2}+M^{2}<0 \text { as } p \rightarrow \infty .
$$

In other words, $\Phi(p, 0)$ and $\frac{\partial \Phi(p, 0)}{\partial x^{5}}$, satisfying the above criterion, form a class of functions which admits a correct formulation of the Cauchy problem for f.e. (3) with respect to the variable $x^{5}$

$$
\begin{gathered}
{\left[\frac{\partial^{2}}{\partial x^{\mathrm{M}} \partial x_{\mathrm{M}}}-\frac{\partial^{2}}{\partial x_{5}^{2}}-M^{2}\right] \Phi\left(x, x^{5}\right)=0,} \\
\left.\Phi\left(x, x^{5}\right)\right|_{x^{5}=0}=\frac{1}{(2 \mathrm{p})^{3 / 2}} \int e^{-i p x} \Phi(p, 0) d^{4} p, \\
\left.\frac{\partial}{\partial x^{5}} \Phi\left(x, x^{5}\right)\right|_{x^{5}=0}= \\
=\frac{1}{(2 \mathrm{p})^{3 / 2}} \int e^{-i p x} \frac{\partial \Phi(p, 0)}{\partial x^{5}} d^{4} p .
\end{gathered}
$$

It should be noted that if we would develop the Euclidean version of QFT, the f.e. would have the form

$$
\left[\frac{\partial^{2}}{\partial x_{n}^{2}}+\frac{\partial^{2}}{\partial x_{5}^{2}}+M^{2}\right] \Phi\left(x, x^{5}\right)=0
$$




$$
n=1,2,3,4
$$

the region $p_{n}^{2}>M^{2}$, would be an analog to $p^{2}+$ $M^{2}<0$ and the condition of correctness of the relevant Cauchy problem for f.e. (10) would require the initial data in the p-representation, $\Phi(p, 0)$ and $\frac{\partial \Phi(p, 0)}{\partial x^{5}}$, to decrease exponentially outside the sphere $p_{n}^{2}=M^{2}$. Thus, the fundamental mass $M$ in some sense should play the role of the cutoff parameter in the ultraviolet region.

\section{Results and discussion}

Let us explain why one should pose the Cauchy problem for f.e. with respect to the coordinate $x^{5}$ and just in the correct formulation. The point is that the Cauchy date $\Phi(p, 0)$ and $\frac{\partial \Phi(p, 0)}{\partial x^{5}}$ are the fields defined in the four-dimensional space-time. Their number depends on the order of the differential equation (8) with respect to the variable $x^{5}$. If the Cauchy problem (8-10) is correct, the f.e. solution is given by the Fourier integral ( 7 ), being unique by construction. Consequently, there is a one-to-one correspondence

$$
\Phi\left(x, x^{5}\right) \leftrightarrow\left(\begin{array}{c}
\Phi(x, 0) \\
\partial \Phi(x, 0) / \partial x^{5}
\end{array}\right)
$$

In other words, the statement that to each field in the 5-space there corresponds its wave function $\Phi\left(x, x^{5}\right)$, obeying f.e. (3), implies that each of these fields in the usual space-time is described by the wave function with a doubled number of components

$$
\left(\begin{array}{c}
\Phi(x, 0) \\
\partial \Phi(x, 0) / \partial x^{5}
\end{array}\right)=\left(\begin{array}{l}
\Phi_{1}(x) \\
\Phi_{2}(x)
\end{array}\right)
$$

We should like to note that having placed the Cauchy problem equation (3) for with respect to the coordinate $x^{5}$ and just in the correct formulation in the basis of QFT, in fact, we have introduced a new concept of the field, which is not equivalent to the notion of the field developed in the theory with constant curvature momentum space.

From here we see that fields are doubled. It appears the field $\Phi_{2}(x)$ participates only in interactions. Due to it there are new members in diagrams. Then, it is natural to assume that the initial data obey the Lagrangian equations of motion following from the action principle

$$
S=\int d^{4} x L\left[\Phi(x, 0), \frac{\partial \Phi(x, 0)}{\partial x^{5}}\right]
$$

The basic problem of the new theory was is to construct explicit expressions for the Lagrangians $L\left[\Phi(x, 0), \frac{\partial \Phi(x, 0)}{\partial x^{5}}\right]$ in physically interesting cases, to clarify the meaning of additional field variables and to extract new physical effects in the region of super-high energies $E \geq M$. Partially, this problem has been solved earlier [3-6, 17-20]. Thus, a doubled number of field degrees of freedom specific of the new scheme disappears as $M \rightarrow \infty$. Hence, specifically,

$$
\begin{gathered}
\lim _{M \rightarrow \infty} L\left[\Phi(x, 0), \frac{\partial \Phi(x, 0)}{\partial x^{5}}\right]= \\
=L[\Phi(x, 0)]
\end{gathered}
$$

Certainly, if in formulating the Cauchy problem we imposed the initial conditions at an arbitrary fixed value

$$
x^{5}=\text { const }
$$

then all our conclusions would be the previous ones and the formulae would undergo trivial changes. For instance, there would appear the following expression for the action

$$
S=\int_{x^{5}=\text { const }} d^{4} x L\left[\Phi\left(x, x^{5}\right), \frac{\partial \Phi\left(x, x^{5}\right)}{\partial x^{5}}\right] .
$$

Thus, we receive actions for scalar, Dirac and vector fields. Basically symmetry of the equation of motion-simultaneously is the symmetry of action. Therefore it is satisfied

$$
\partial S / \partial x^{5}=0
$$

Let's consider for a neutral scalar field $\Phi\left(x, x^{5}\right)$ particles with zero mass. According to

$$
\Phi\left(x, x^{5}\right) \simeq e^{i M x^{5}} \Phi(x, 0)
$$

in the $\operatorname{limit} M \rightarrow \infty$

$$
\varphi\left(x, x^{5}\right) \simeq e^{i M x^{5}} \varphi(x, 0)
$$

The choice of a solution of f.e. in the form of (13) corresponds to the following initial data $(5,6)$

$$
\varphi\left(x, x^{5}\right) \equiv \varphi(x)
$$




$$
-\frac{i}{M} \frac{\partial \varphi\left(x, x^{5}\right)}{\partial x^{5}} \equiv X(x)
$$

We can conclude that of the two field functions $\varphi(x)=\varphi(x, 0)$ and $X(x) \equiv-\frac{i}{M} \frac{\partial \varphi(x, 0)}{\partial x^{5}}$ used in the new formalism to describe a massless neutral scalar field only the first $\varphi(x)$ is physical since only it has a fully adequate equation of motion. The field $५(x)$ is pure auxiliary. It has no its own equation of motion and its values fully depend on its values of the field $\varphi(x)$.

Maybe, in future $X(x) \equiv-\frac{i}{M} \frac{\partial \varphi(x, 0)}{\partial x^{5}}$ will be interpreted as a candidate on the "Phantom field", responsible for acceleration of expansion the Universe.

Thus, the action for the field of no interacting neutral scalar particles with zero mass has in our approach the following form:

$$
\begin{gathered}
S=\frac{1}{2} \int d^{4} x\left[\frac{\partial \varphi\left(x, x^{5}\right)}{\partial x_{\mathrm{M}}} \frac{\partial \varphi\left(x, x^{5}\right)^{+}}{\partial x_{\mathrm{M}}}+\left|-i \frac{\partial \varphi\left(x, x^{5}\right)}{\partial x^{5}}-M \varphi\left(x, x^{5}\right)\right|^{2}\right]= \\
=\frac{1}{2} \int d^{4} x\left[\frac{\partial \varphi(x)}{\partial x_{\mathrm{M}}} \frac{\partial \varphi(x)^{+}}{\partial x_{\mathrm{M}}}+M^{2}(X(x)-\varphi(x))^{2}\right] .
\end{gathered}
$$

This expression satisfies the correspondence principle with the standard theory since at $M \rightarrow \infty$ by virtue of $\varphi\left(x, x^{5}\right) \simeq e^{i M x^{5}} \varphi(x, 0)$

$$
S=\frac{1}{2} \int d^{4} x \frac{\partial \varphi(x)}{\partial x_{\mathrm{M}}} \frac{\partial \varphi(x)^{+}}{\partial x_{\mathrm{M}}}
$$

The action of the free Dirac field can be written also in the configuration 5-space:

$$
\begin{gathered}
S=\frac{1}{2} \int d^{4} x\left\{\bar{\Psi}\left(x, x^{5}\right)(i \hat{\partial}+M)\left(-\frac{i}{M} \frac{\partial}{\partial x^{5}} \Psi\left(x, x^{5}\right)\right)+\overline{\left(-\frac{i}{M} \frac{\partial}{\partial x^{5}} \Psi\left(x, x^{5}\right)\right)}(i \hat{\partial}+M) \Psi\left(x, x^{5}\right)-\right. \\
\left.-M \overline{\left(-\frac{i}{M} \frac{\partial}{\partial x^{5}} \Psi\left(x, x^{5}\right)\right)}\left(-\frac{i}{M} \frac{\partial}{\partial x^{5}} \Psi\left(x, x^{5}\right)\right)-\bar{\Psi}\left(x, x^{5}\right)\left(M+\frac{(i \partial)^{2}}{M^{2}}\right) \Psi\left(x, x^{5}\right)\right\},
\end{gathered}
$$

where the spinor field $\psi\left(x, x^{5}\right)$ satisfies f.e. (3). According to our general concept, $\psi(p)$ and $\varphi(p)$ are Fourier transforms of the initial data

$$
\Psi(x, 0)=\Psi(x),-\frac{i}{M} \frac{\partial}{\partial x^{5}} \Psi(x, 0) \equiv X(x)
$$

sufficiently decreasing in the region

$$
p^{2}+M^{2}<0 \text { as }|p| \rightarrow \infty .
$$

The same procedure, we applied in the scalar and spinor cases, leads to the following total action integral of the electromagnetic field in QFT with the fundamental mass

$$
S=-\frac{1}{4} \int d^{4} x\left\{\begin{array}{c}
\mathrm{F}_{\mathrm{KL}}\left(x, x^{5}\right) F^{K L}\left(x,-x^{5}\right)+ \\
+2\left|\frac{\partial A^{\mathrm{M}}\left(x, x^{5}\right)}{\partial x^{5}}-i M \mathrm{~A}_{5}\left(x, x^{5}\right)-\frac{\partial \mathrm{A}_{5}\left(x, x^{5}\right)}{\partial x^{5}}\right|^{2}
\end{array}\right\}
$$

where

$$
\mathrm{F}_{\mathrm{KL}}\left(x, x^{5}\right)=\frac{\partial}{\partial x^{K}}\left(e^{-i M x^{5}} \mathrm{~A}_{\mathrm{L}}\left(x, x^{5}\right)\right)-\frac{\partial}{\partial x^{L}}\left(e^{-i M x^{5}} \mathrm{~A}_{\mathrm{K}}\left(x, x^{5}\right)\right) \quad L, K=0,1,2,3,5 .
$$


The Lagrangian density in (21) is not a pure local expression in the configurational 5-space but a quantity invariant under local gauge transformations of the 5-potential

$$
\begin{aligned}
& e^{-i M x^{5}} \mathrm{~A}_{\mathrm{K}}\left(x, x^{5}\right) \rightarrow e^{-i M x^{5}} \mathrm{~A}_{\mathrm{K}}\left(x, x^{5}\right)- \\
& -\frac{\partial}{\partial x^{K}}\left(e^{-i \text { 滥 } x^{5}} \Lambda\left(x, x^{5}\right)\right) \\
& K=0,1,2,3,5 .
\end{aligned}
$$

where the function $\Lambda\left(x, x^{5}\right)$, like $\mathrm{A}_{\mathrm{K}}\left(x, x^{5}\right)$, obeys f.e. (3). Naturally as usual,

$$
\partial S / \partial x^{5}=0 .
$$

Could the advanced theory be free from ultraviolet divergences? At the present we do not have the final answer what this issue is concerned, however, we can calculate effective cross sections of some processes which are in good agreement with experiments, and this allows to estimate the contribution of fundamental mass. Research in building consecutive new QFT with fundamental weight are resulted also in publications $[13,14,15$, $16]$.

\section{Conclusions}

Further, we investigated the phenomenological (experimental) consequences of such a quantum field theoretical model $[17,18,19,20]$. Calculations of cross sections corresponding to various basic processes are executed to the second order. On the basis of the QFT with FM, calculations of cross sections for processes such as $e^{-} e^{-} \rightarrow e^{-} e^{-}$, $e^{-} e^{+} \rightarrow e^{-} e^{+}$and $e^{-} e^{+} \rightarrow \mathrm{M}^{-} \mathrm{M}^{+}$have been carried out by taking into account the polarization of particles. Is estimated contribution FM to crosssection sections. Some experimental consequences are predicted. In all these calculations the polarization of particles can be taken into account.

\section{References}

1. V. G. Kadyshevsky. On the theory of quantized space-time // JETF. - 1961. - Vol. 41. -No.6. - P.1885-1894 (in Russian).

2. V.G. Kadyshevsky. Fundamental length hypothesis and new concept of Gauge vector field // Nuclear Physics B. - 1978. - Vol. 141. - No. 4. - P. 477-496.

3. W. Heisenberg. Introduction to the unified field theory of elementary particles. // Iters. Publ. UK, - 1966. $192 \mathrm{p}$.

4. C.N. Yang. On quantized space-time // Phys. Rev. - 1947. -Vol. 72. - P.874.

5. A.A. Komar, M.A. Markov. An example of a field theory with indefinite metric in Hilbert space II // Nucl. Phys. - 1959. - Vol. 12. - P.190. $701 \mathrm{p}$.

6. J. D. Bjorken. Identification of elementary forces and Gauge theories. Harwood Academic Publisher. - 1979. -

7. M.V. Chizhov, A.D. Donkov, R.M. Ibadov, V.G. Kadyshevsky, M.D. Mateev. Quantum field theory and a new universal high energy scale Dirac fields // Nuovo Cimento. - 1985. - Vol. 87A. - No.3. - P.350.

8. M.V. Chizhov, A.D. Donkov, R.M. Ibadov, V.G. Kadyshevsky, M.D. Mateev. Quantum field theory and a new universal high energy scale. Gauge vector fields // Nuovo Cimento. - 1985. - Vol. 87A. - No.4. - P.375.

9. R.M. Ibadov, V.G. Kadyshevsky. New formulation of quantum field theory with fundamental mass // Proceedings 5th International Symposium on Selected Topics in Statistical Mechanics, Dubna, World Scientific, Singapore. - 1989. - P. 131.

10. R.M. Ibadov, V.G. Kadyshevsky. About transformations of supersymmetry in theories of a field with fundamental mass // Preprint JINR. - 1986. -Vol. 86. - P. 4.

11. R.M. Ibadov, V.G. Kadyshevsky. The new point of view on auxiliary fields in supersymmetric models // Works of VIII International Meeting on problems of the Quantum Theory of a Field. Alushta. Dubna, - 1988. - Vol. D2-87-798. - P.141;

12. R. M. Ibadov, V. G. Kadyshevsky. On supersymmetry transformations in the field theory with a fundamental mass // JINR Preprint. - 1986. - Vol. R2-86-835.

13. V. G. Kadyshevsky, M. D. Mateev, V. N. Rodionov, A. S. Sorin. Towards a geometric approach to the formulation of the standard model // Dokl. Phys, 51. - 2006. - P. 287.

14. V.G. Kadyshevsky, M.D. Mateev, V.N. Rodionov, A.S. Sorin. Towards a maximal mass model // arXiv: 0708.4205v1[help-ph]. E-print. - 2007. - Vol. 150. - P. 1-28. 
15. V.G. Kadyshevsky, M.D. Mateev, V.N. Rodionov, A.S. Sorin. Towards a geometric approach to the formulation of the standard model // Dokl.Phys. - 2006. - Vol. 51. - P. 287-290.

16. V. N. Rodionov, G. A. Kravtsova. An algebraic PT-symmetric quantum theory with a maximal mass // Physics of Particles and Nuclei. -2016. - Vol. 47. - No. 2. - P. 135-156.

17. A.D. Donkov, R.M. Ibadov, V.G. Kadyshevsky, M.D. Mateev, M.V. Chizhov. Some experimental consequences of a hypothesis about fundamental length // Izvestiya AN USSR, Series Physics. - 1982. -Vol. 46. - No. 9. - P.1772.

18. R.M. Ibadov, M.V. Chizhov. Application of quantum electrodynamics with in the fundamental length to high power process // Izvestiya AN UZSR, Series Physics and Mathematics. - 1983. -Vol. 5. - P.38.

19. R.M. Ibadov. Deep elastic scattering of electron in nucleons and fundamental lengths // Izvestiya AN UZSR, Series Physics and Mathematics, - 1984. - Vol. 3. - P.44.

20. R. Ibadov, S. Tuhtamishev, U. Khodjaeva. Some experimental consequences hypotheses about fundamental mass // European Science. - 2017. -Vol. 24. - No.2. - P. 6-13. 